\title{
Meta-analysis of the relationship between $p 21$ Ser31Arg polymorphism and lung cancer susceptibility
}

\author{
G. Lin*, F. Fang*, X.-J. Yu and L. Yu \\ State Key Laboratory of Genetic Engineering, Institute of Genetics, \\ School of Life Sciences, Fudan University, Shanghai, P.R. China \\ *These authors contributed equally to this study. \\ Corresponding author: L. Yu \\ E-mail: longyu@fudan.edu.cn
}

Genet. Mol. Res. 10 (4): 2449-2456 (2011)

Received October 22, 2010

Accepted May 2, 2011

Published October 13, 2011

DOI http://dx.doi.org/10.4238/2011.October.13.2

\begin{abstract}
The cyclin-dependent kinase inhibitor 1A (also known as $p 21$ ) is thought to be involved in tumor development by mediating cell cycle arrest through the inhibition of cyclin/CDK activity. To explore the relationship of Ser31Arg polymorphism in the $p 21$ gene with the risk of developing lung cancer, we performed an overall and stratified meta-analysis based on ethnicity, lung cancer subtypes and source of controls, with six eligible studies (2366 cases and 3320 controls). No significant variation in lung cancer risk was detected in any of the genetic models in the overall, and the ethnicity-based and cancer subtype-based subgroup analyses. However, in the subgroup analysis based on source of controls, significant opposite associations were observed; a significantly increased lung cancer risk was observed in the hospital-based control subgroup, while a significantly decreased lung cancer risk was detected in the mixed-source control and unknown-source
\end{abstract}


control subgroups. In summary, based on our meta-analysis, $p 21$ Ser31Arg polymorphism does not appear to act as an independent lung cancer risk factor and is more likely to act together with other genetic and non-genetic factors in the development of lung cancer; this needs further investigation.

Key words: $p 21$; Ser31Arg; Polymorphism; Lung cancer risk; Meta-analysis

\section{INTRODUCTION}

Lung cancer is a common cancer all over the world with high mortality rate, and current knowledge of the molecular basis of lung cancer susceptibility is limited (Kiyohara et al., 2002; Mitsudomi, 2010). The cyclin-dependent kinase inhibitor 1A ( 221$)$ gene is a well-studied gene encoding a protein that inhibits the activity of the cyclin/CDK family members and promotes cell cycle arrest under multiple stimulations (Xiong et al., 1993; Harada and Ogden, 2000; Abbas and Dutta, 2009). A single nucleotide polymorphism has been reported at codon 31 of the $p 21$ gene (Ser31Arg, rs1801270) with an AGC (Ser) to AGA (Arg) change (Chedid et al., 1994). To date, several case-control studies have investigated the association between $p 21$ Ser31 Arg polymorphism and lung cancer risk. However, with relatively small sample sizes, these former studies provided limited information and could not draw a convincing conclusion. Hence, we performed a meta-analysis based on 6 eligible studies (2366 cases and 3320 controls in total), with the intention of obtaining a more reliable lung cancer risk assessment in association with $p 21 \mathrm{Ser} 31 \mathrm{Arg}$ polymorphism.

\section{MATERIAL AND METHODS}

The workflow of this study is illustrated in Figure 1.

\section{Literature exploration, selection and data collection}

In this study, we searched for papers published before August 9, 2010 in four widely used databases (PubMed, Web of Science, EBSCO, and CNKI), with key words "p21"/“CDKN1A"/“Cip1"/“WAF1", "polymorphism" and "lung". The papers obtained were further screened for the meta-analysis based on the following selection criteria: i) full-text English-written study, ii) study providing complete case and control data about the relationship between $p 21$ Ser31Arg polymorphism and lung cancer risk, iii) studies using overlapped sample of cases and controls. The selected papers were compared, and the most complete studies from them were included in our meta-analysis.

In this study, two investigators independently collected data from each eligible paper to reduce bias in data collection. The data consisted of the first author, year published, country of origin, ethnicity, source of controls, lung cancer subtypes, and numbers of cases and controls with the Ser/Ser, Ser/Arg, and Arg/Arg genotypes. Through checking and consultation between the two investigators, a final data collection was determined. 


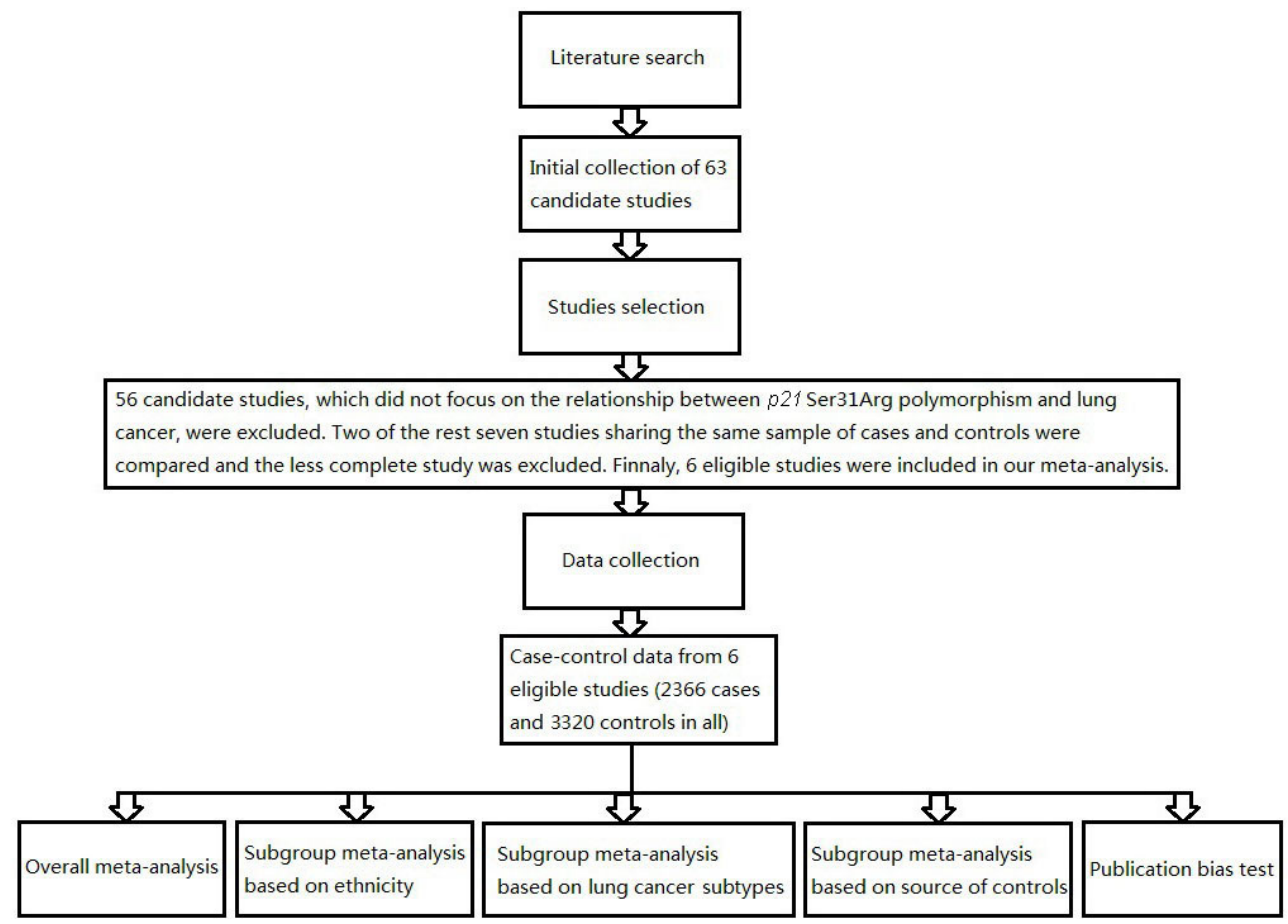

Figure 1. Workflow chart of this study.

\section{Meta-analysis methods}

According to the data collected from each paper included, we performed both an overall meta-analysis and a subgroup meta-analysis based on ethnicity, lung cancer subtypes, and source of controls, to evaluate the relationship between $p 21$ Ser31Arg polymorphism and lung cancer risk. In the overall as well as the subgroup meta-analysis, pooled odds ratios (ORs) and 95\% confidence intervals (CIs) for Ser/Ser versus Arg/Arg, Ser/Arg versus Arg/Arg, Ser/Ser + Ser/Arg versus Arg/Arg, and Ser/Ser versus Ser/Arg + Arg/Arg were all calculated by the fixed-effects model or random-effects model. The model was chosen based on the heterogeneity test, and therefore, we used the $\chi^{2}$-based $Q$-test in this study (Lau et al., 1997). When the $Q$-test reported a $P$ value of more than 0.10 , the fixed-effects model was used to calculate the pooled ORs (Mantel and Haenszel, 1959); the random-effects model was used otherwise (DerSimonian and Laird, 1986).

Publication bias was also tested using the Begg funnel plot and the Egger test (Egger et al., 1997). If the funnel plot was asymmetric and if the Egger test reported a P value of less than 0.05 , publication bias was assumed to exist.

In this study, we used the Stata software version 10.0 (Stata Corporation, College Station, TX, USA) to carry out the meta-analysis. 


\section{RESULTS}

\section{Studies and data included in this meta-analysis}

After searching, 63 candidate studies were collected and 6 eligible studies were eventually determined for meta-analysis (Sjalander et al., 1996; Shih et al., 2000; Hsia et al., 2003; Su et al., 2003; Popanda et al., 2007; Choi et al., 2008). The six studies included, providing 2366 cases and 3320 controls, varied with respect to ethnicity (3 studies in Asians and 3 studies in Caucasians), and source of controls (4 studies having hospitalbased controls, 1 having mixed sources, and 1 having unknown source). Of the 6 eligible studies, 5 used cases with mixed lung cancer subtypes. The control groups of the 6 eligible studies were all in Hardy-Weinberg equilibrium (HWE) $(\mathrm{P}>0.05)$. The information from these 6 studies and the numbers of cases and controls with Ser/Ser, Ser/Arg, and Arg/Arg genotypes reported in each study are all presented in Table 1.

\section{Overall and subgroup meta-analysis results}

In this study, we performed both an overall meta-analysis and subgroup metaanalysis based on ethnicity, lung cancer subtypes, and source of controls, and the detailed results of our meta-analysis are shown in Table 2A,B and Figure 2.

The results of the overall meta-analysis indicated the lack of correlation between p21 Ser31 Arg polymorphism and lung cancer risk for all genetic models $(\mathrm{OR}=1.11$, $95 \% \mathrm{CI}=0.85-1.45$ for $\mathrm{Ser} / \mathrm{Ser}$ versus $\mathrm{Arg} / \mathrm{Arg} ; \mathrm{OR}=0.94,95 \% \mathrm{CI}=0.74-1.20$ for $\mathrm{Ser} /$ Arg versus Arg/Arg; OR $=0.99,95 \% \mathrm{CI}=0.79-1.25$ for Ser/Ser + Ser/Arg versus Arg/ $\mathrm{Arg}$, and $\mathrm{OR}=1.03,95 \% \mathrm{CI}=0.82-1.28$ for Ser/Ser versus Ser/Arg $+\mathrm{Arg} / \mathrm{Arg}$; see Table $2 \mathrm{~A}$ and Figure 2).

Similarly, in the ethnicity-based subgroup meta-analysis, no obvious association for all genetic models was found either in Asians $(\mathrm{OR}=1.10,95 \% \mathrm{CI}=0.82-1.46$ for Ser/ Ser versus Arg/Arg; $\mathrm{OR}=0.89,95 \% \mathrm{CI}=0.52-1.52$ for $\mathrm{Ser} / \mathrm{Arg}$ versus $\mathrm{Arg} / \mathrm{Arg} ; \mathrm{OR}=0.92$, $95 \% \mathrm{CI}=0.59-1.46$ for $\mathrm{Ser} / \mathrm{Ser}+\mathrm{Ser} / \mathrm{Arg}$ versus Arg/Arg, and $\mathrm{OR}=1.14,95 \% \mathrm{CI}=0.91-$ 1.43 for Ser/Ser versus Ser/Arg + Arg/Arg; see Table 2A and Figure 2) or in Caucasians $(\mathrm{OR}=1.20,95 \% \mathrm{CI}=0.58-2.50$ for $\mathrm{Ser} / \mathrm{Ser}$ versus $\mathrm{Arg} / \mathrm{Arg} ; \mathrm{OR}=1.06,95 \% \mathrm{CI}=0.50-2.24$ for Ser/Arg versus Arg/Arg; OR = 1.18, 95\%CI = 0.57-2.44 for Ser/Ser + Ser/Arg versus $\mathrm{Arg} / \mathrm{Arg}$, and $\mathrm{OR}=0.94,95 \% \mathrm{CI}=0.61-1.44$ for Ser/Ser versus Ser/Arg $+\mathrm{Arg} / \mathrm{Arg}$; see Table 2A and Figure 2).

In addition, a significant association between $p 21$ Ser31Arg polymorphism and lung cancer risk was not observed in the subgroup meta-analysis based on lung cancer subtypes for all genetic models either in squamous cell carcinoma $(\mathrm{OR}=1.08,95 \% \mathrm{CI}=$ 0.50-2.32 for Ser/Ser versus Arg/Arg; OR $=1.20,95 \% \mathrm{CI}=0.60-2.39$ for Ser/Arg versus $\mathrm{Arg} / \mathrm{Arg} ; \mathrm{OR}=1.17,95 \% \mathrm{CI}=0.61-2.25$ for Ser/Ser $+\mathrm{Ser} / \mathrm{Arg}$ versus $\mathrm{Arg} / \mathrm{Arg}$, and $\mathrm{OR}=$ $1.10,95 \% \mathrm{CI}=0.75-1.61$ for Ser/Ser versus Ser/Arg $+\mathrm{Arg} / \mathrm{Arg}$; see Table 2B) or in adenocarcinoma $(\mathrm{OR}=1.25,95 \% \mathrm{CI}=0.59-2.64$ for $\mathrm{Ser} / \mathrm{Ser}$ versus $\mathrm{Arg} / \mathrm{Arg} ; \mathrm{OR}=1.65,95 \% \mathrm{CI}$ $=0.85-3.22$ for Ser/Arg versus $\mathrm{Arg} / \mathrm{Arg} ; \mathrm{OR}=1.49,95 \% \mathrm{CI}=0.79-2.84$ for Ser $/ \mathrm{Ser}+\mathrm{Ser} /$ Arg versus Arg/Arg, and $\mathrm{OR}=0.81,95 \% \mathrm{CI}=0.57-1.14$ for $\mathrm{Ser} / \mathrm{Ser}$ versus $\mathrm{Ser} / \mathrm{Arg}+\mathrm{Arg} /$ Arg; see Table 2B). 


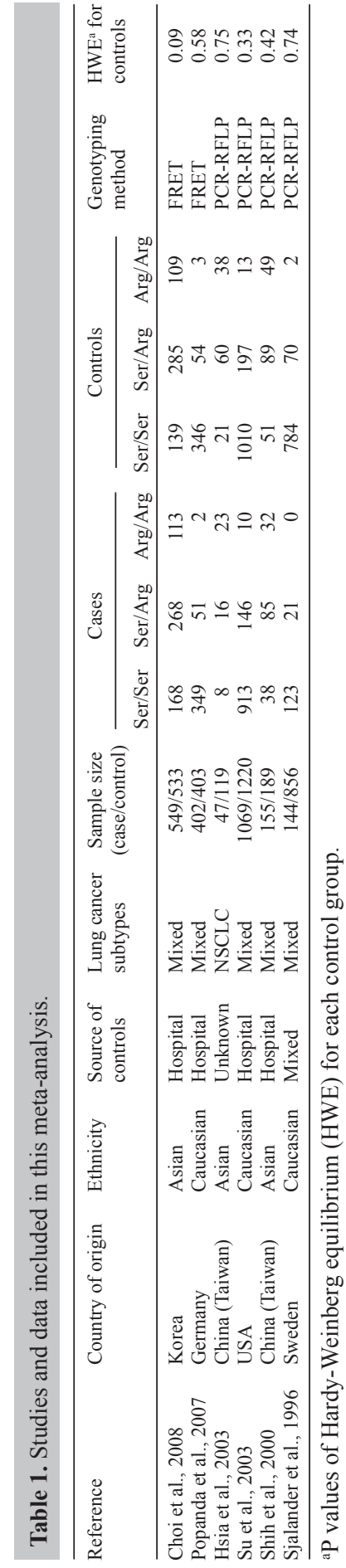

Genetics and Molecular Research 10 (4): 2449-2456 (2011)

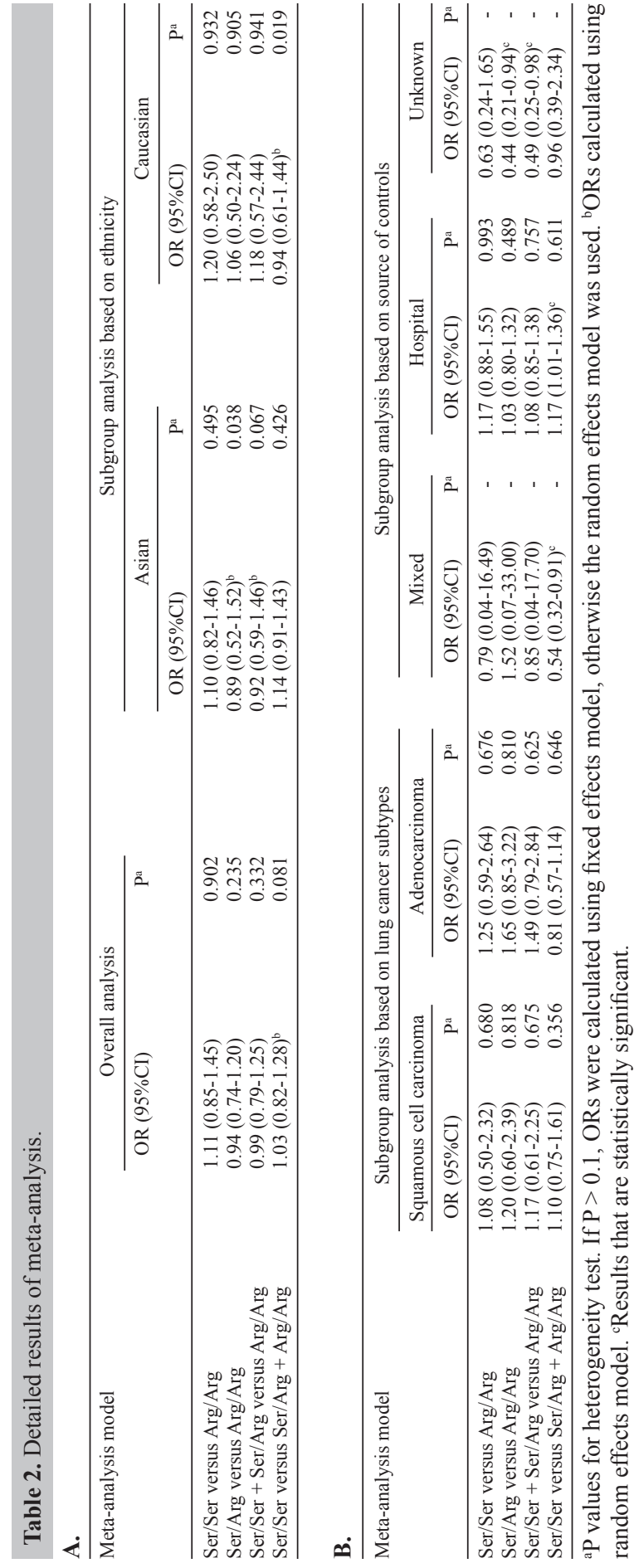

CFUNPEC-RP www.funpecrp.com.br 


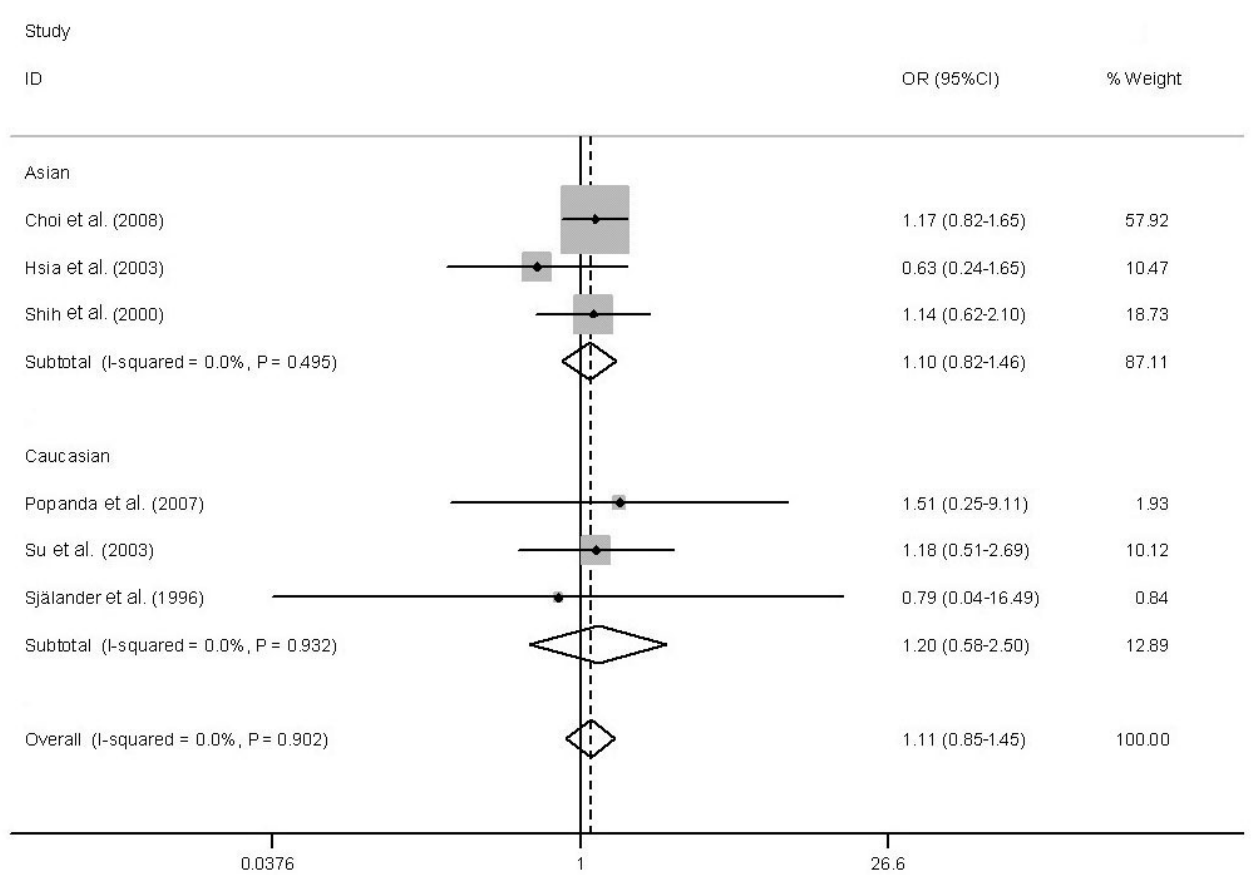

Figure 2. Forest plot for Ser/Ser versus Arg/Arg of the overall meta-analysis and subgroup meta-analysis based on ethnicity using the fixed-effects model.

However, the results of the subgroup analysis based on source of controls were complicated (Table 2B). In this subgroup analysis, a significantly increased lung cancer risk for Ser/Ser versus Ser/Arg + Arg/Arg existed when only hospital-collected controls were used $(\mathrm{OR}=1.17,95 \% \mathrm{CI}=1.01-1.36$; see Table $2 \mathrm{~B})$; while obviously decreased lung cancer risks were found in the mixed-source control subgroup for Ser/Ser versus Ser/Arg + Arg/Arg (OR $=0.54,95 \% \mathrm{CI}=0.32-0.91$; see Table $2 \mathrm{~B}$ ), and in the unknown-source control subgroup for Ser/Arg versus Arg/Arg $(\mathrm{OR}=0.44,95 \% \mathrm{CI}=0.21-0.94$; see Table $2 \mathrm{~B})$ and Ser/Ser + Ser/Arg versus $\mathrm{Arg} / \mathrm{Arg}(\mathrm{OR}=0.49,95 \% \mathrm{CI}=0.25-0.98$; see Table $2 \mathrm{~B})$. HWE tests were performed in this study for control groups of all 6 eligible studies, and every control group was in HWE. Hence, we suspect that the opposite results in the subgroup meta-analysis, based on the source of controls, might have been due to the limited sample size or different population structure. It is still likely that the $p 21$ Ser31Arg polymorphism has no association with lung cancer susceptibility as an independent risk factor.

\section{Publication bias test results}

The results of the Begg funnel plot (plots not shown) and the Egger test showed no publication bias for Ser/Ser versus $\operatorname{Arg} / \operatorname{Arg}(\mathrm{P}=0.530)$, Ser/Arg versus $\operatorname{Arg} / \operatorname{Arg}(\mathrm{P}=0.925)$, $\mathrm{Ser} / \mathrm{Ser}+\mathrm{Ser} / \operatorname{Arg}$ versus $\mathrm{Arg} / \operatorname{Arg}(\mathrm{P}=0.926)$, and Ser/Ser versus Ser/Arg $+\operatorname{Arg} / \operatorname{Arg}(\mathrm{P}=$ $0.122)$ in the overall meta-analysis. 


\section{DISCUSSION}

In this study, the results of our overall meta-analysis did not show any significant association between $p 21$ Ser31Arg polymorphism and lung cancer risk. Our subgroup metaanalyses based on ethnicity and lung cancer subtypes both indicated that the $p 21$ Ser31Arg polymorphism did not act as a lung cancer risk factor directly. These results are in accordance with Chedid et al. (1994) who reported that there was no association between $p 21$ Ser31Arg polymorphism and loss of tumor suppressor activity demonstrated by transfection studies. Therefore, it is probably biologically reasonable to speculate that the $p 21 \mathrm{Ser} 31 \mathrm{Arg}$ polymorphism has no independent association with lung cancer susceptibility.

Moreover, our subgroup meta-analysis based on source of controls revealed a more complicated result: a significantly increased lung cancer risk was observed in the hospitalbased control subgroup; on the contrary, significantly decreased lung cancer risks were detected in both the mixed-source control and the unknown-source control subgroups. The fact that $p 21$ Ser31Arg polymorphism plays opposite roles in subgroups with different sources of controls suggests the potential influence of the control sources on the outcomes. The results, however, should be considered prudently since a few limitations may skew the outcomes. Due to insufficient sample sizes of the studies included (for example, only one eligible study belonged to the mixed-source control subgroup and only one to the unknown-source control subgroup), further investigation with larger sample size is necessary. Besides, more detailed individual data such as age, gender, and lifestyle (for example, smoking status) may also need to be assessed, and the confounding influence of $p 53$ status and other $p 21$ polymorphisms should also be considered.

In conclusion, the previously reported controversial conclusions on the correlation of p21 Ser31Arg polymorphism with lung cancer susceptibility may arise from statistical bias, such as limited sample size or some methodological errors. As suggested by our meta-analysis with a total of 6 eligible studies (2366 cases and 3320 controls in all), p21 Ser31Arg polymorphism does not appear to act as an independent risk factor of developing lung cancer, and further studies are needed to determine its contribution to the genesis of lung cancer interacting with other determinants.

\section{ACKNOWLEDGMENTS}

Research supported by National 973 programs of China (grant \#2004CB518605) and the National 863 project of China (grant \#2006AA020501).

\section{REFERENCES}

Abbas T and Dutta A (2009). p21 in cancer: intricate networks and multiple activities. Nat. Rev. Cancer 9: 400-414.

Chedid M, Michieli P, Lengel C, Huppi K, et al. (1994). A single nucleotide substitution at codon 31 (Ser/Arg) defines a polymorphism in a highly conserved region of the p53-inducible gene WAF1/CIP1. Oncogene 9: 3021-3024.

Choi YY, Kang HK, Choi JE, Jang JS, et al. (2008). Comprehensive assessment of P21 polymorphisms and lung cancer risk. J. Hum. Genet. 53: 87-95.

DerSimonian R and Laird N (1986). Meta-analysis in clinical trials. Control Clin. Trials 7: 177-188.

Egger M, Davey SG, Schneider M and Minder C (1997). Bias in meta-analysis detected by a simple, graphical test. BMJ 315: 629-634.

Harada K and Ogden GR (2000). An overview of the cell cycle arrest protein, p21(WAF1). Oral Oncol. 36: 3-7.

Hsia TC, Chiang HC, Chiang D, Hang LW, et al. (2003). Prediction of survival in surgical unresectable lung cancer by 
artificial neural networks including genetic polymorphisms and clinical parameters. J. Clin. Lab. Anal. 17: 229-234. Kiyohara C, Otsu A, Shirakawa T, Fukuda S, et al. (2002). Genetic polymorphisms and lung cancer susceptibility: a review. Lung Can. 37: 241-256.

Lau J, Ioannidis JP and Schmid CH (1997). Quantitative synthesis in systematic reviews. Ann. Intern. Med. 127: 820-826.

Mantel N and Haenszel W (1959). Statistical aspects of the analysis of data from retrospective studies of disease. J. Natl. Cancer Inst. 22: 719-748.

Mitsudomi T (2010). Advances in target therapy for lung cancer. Jpn. J. Clin. Oncol. 40: 101-106.

Popanda O, Edler L, Waas P, Schattenberg T, et al. (2007). Elevated risk of squamous-cell carcinoma of the lung in heavy smokers carrying the variant alleles of the TP53 Arg72Pro and p21 Ser31Arg polymorphisms. Lung Cancer 55: 25-34.

Shih CM, Lin PT, Wang HC, Huang WC, et al. (2000). Lack of evidence of association of p21WAF1/CIP1 polymorphism with lung cancer susceptibility and prognosis in Taiwan. Jpn. J. Cancer Res. 91: 9-15.

Sjalander A, Birgander R, Rannug A, Alexandrie AK, et al. (1996). Association between the p21 codon 31 A1 (arg) allele and lung cancer. Hum. Hered. 46: 221-225.

Su L, Liu G, Zhou W, Xu LL, et al. (2003). No association between the p21 codon 31 serine-arginine polymorphism and lung cancer risk. Cancer Epidem. Biomar. Prev. 12: 174-175.

Xiong Y, Hannon GJ, Zhang H, Casso D, et al. (1993). p21 is a universal inhibitor of cyclin kinases. Nature 366: 701-704. 